Kinderzahnheilkunde

\section{Bulkfüll-Komposit bietet schnellere Füllungstherapie von Milchzähnen}

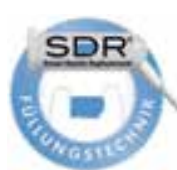

Neben der Vielzahl bisheriger Indikationen ist SDR (Smart Dentin Replacement) von DENTSPLY jetzt in der Kinderzahnheilkunde für die Versorgung von Zähnen der 1. Dentition zugelassen. Das BulkfüllKomposit ist kompatibel zu sämtlichen methacrylatbasierten Adhäsiven. In der Kinderzahnheilkunde empfiehlt sich besonders die Kombination mit dem auf SDR abgestimmten Universal-Adhäsiv Xeno Select, welches optional in der schnelleren Self-Etch-Technik verwendet werden kann.
Eine Vielzahl materialwissenschaftlicher und klinischer Studien belegt die Vorteile dieses fließfähigen selbstnivellierenden Bulkfüll-Materials. Mit der jetzt vorliegenden Indikation für die Versorgung kariöser Läsionen der 1. Dentition steht nun auch Kinderzahnärzten die zeitsparenden Bulkfüll-Technik zur Verfügung. Ein weiterer Pluspunkt: Bei der Füllungstherapie von Milchzähnen mit dem Material ist in der Regel ein Abschluss mit einem Deckkomposit entbehrlich. Dass SDR für außergewöhnlich hohe klinische
Sicherheit steht, beweist seine große Indikationsbreite, die weit über die Kinderzahnheilkunde hinaus reicht: Dentinersatz bei Klasse I und II, Einsatz als Liner, Stumpfaufbauten, Postendo-Verschluss, Fissurenversiegelung sowie kleine KlasseI-Füllungen. Optimal ergänzt wird SDR durch das Teilmatrizensystem Palodent Plus, das nanokeramische Komposit Ceram.X sowie das zertifizierte DENTSPLY Endo-Resto-System.

Weitere Informationen zu SDR sind unter der kostenlosen DENTSPLY Service-Line für Deutschland 080 00/73 5000 erhältlich oder unter www.dentsply.de/SDR.

Nach einer Pressemitteilung der Dentsply DeTrey, Konstanz 